\title{
Estudio piloto comparativo de dos soluciones de sellado en catéteres de hemodiálisis: heparina sódica al $5 \%$ frente a fibrilin ${ }^{\circledR}$
}

\author{
Constantino Rivera Fernández ${ }^{*}$, Lucía Lamela Rivas ${ }^{* *}$, Carmen Ornosa Agra*
}

Nefrólogo*, Enfermeras**, Unidad de Hemodiálisis Hospital Quirón. A Coruña

\section{Resumen}

El sellado de catéteres en hemodiálisis suele ser motivo de controversia entre los distintos profesionales dedicados a la hemodiálisis.

El objetivo del presente estudio es comparar dos soluciones de sellado de catéter para hemodiálisis: heparina al $5 \%$ y fibrilin (heparina $20 \mathrm{ui} / \mathrm{ml}+$ metil y propilparaben).

Estudiamos 8 pacientes (mujeres) de $69 \pm 12$ años portadoras de catéter tunelizado de $17 \pm 7$ meses de duración del catéter, estables y que habían dado su consentimiento para el estudio. Inicialmente se selló con fibrilin durante un mes ( 12 sesiones) y posteriormente con heparina al $5 \%$ el mismo periodo de tiempo. Se registró velocidad de bomba, flujo efectivo, presión venosa, $\mathrm{KT}$, necesidad de utilizar fibrinolíticos, infecciones, $\mathrm{n}^{\circ}$ de manipulaciones, hipotensiones, recirculación, KT/V, TP, TPTA. Se compararon los estudios mediante $t$ student .

Después de dos meses de estudio se observó mayor flujo efectivo $318 \pm 23 \mathrm{ml} / \mathrm{m}$ en catéteres sellados con heparina frente a $307 \pm 17 \mathrm{ml} / \mathrm{m}$ con fibrilin $(p=$ 0,008 ), menor presión venosa $147 \pm 12 \mathrm{~mm} \mathrm{Hg}$ en heparina frente a $168 \pm 17$ en fibrilin $(p=0,006)$, mayor KT en heparina $43 \pm 3$ litros frente a $41 \pm 4$ litros en fibrilin. A pesar de estas mejores condiciones, clínicamente no supusieron diferencias en

\section{Correspondencia: \\ Lucía Lamela Rivas \\ Unidad de Hemodiálisis \\ Hospital Quirón de A Coruña \\ Calle Londres n². 15009 A Coruña \\ E-mail: lucyalr@hotmail.com}

la eficacia dialítica KT/V heparina $1,56 \pm 0,2$ frente a $1,59 \pm 0,2$ en fibrilin. Si se observó un mayor $n^{\circ}$ de manipulaciones del catéter en heparina $12 \pm 0,2$ frente a $9,4 \pm 1,3$ en fibrilin $(p=0,001)$. No existieron diferencias en aparición de infecciones, recirculación, necesidad de fibrinoliticos 0 alteraciones de coagulación.

Concluimos que el sellado de catéteres de Hemodiálisis con Fibrilin es una alternativa eficaz a la heparina al $5 \%$. No se acompaña de un mayor grado de disfunción del catéter y si de un menor $n^{\circ}$ de manipulaciones, lo que podría condicionar un menor $n^{0}$ de infecciones asociado a catéteres.

\section{PALABRAS CLAVE:}

- SELLADO

- CATÉTER

- HEPARINA 5\%

- FIBRILIN

Comparative pilot study of two haemodialysis catheter lock solutions: $5 \%$ heparin sodium compared to fibrilin $\circledast$

\section{Abstract}

Catheter locks in haemodialysis are usually a source of controversy among the different professionals involved in haemodialysis.

The aim of this study is to compare two haemodialysis catheter lock solutions: $5 \%$ heparin and fibrilin (heparin 20ui/ml + methyl and propyl paraben). 
We studied 8 patients (women) aged $69 \pm 12$ years with tunnelled catheters of $17 \pm 7$ months' duration, whose condition was stable and who had given their consent to the study. Initially, the fibrilin lock solution was used with the catheters for one month ( 12 sessions) and then $5 \%$ heparin for the same length of time. Pump speed, effective flow, venous pressure, $K T$, the need to use fibrinolytics, infections, number of handlings, hypotension episodes, recirculation, KT/V, TP, TPTA were measured. The results were compared using student's t-test.

After two months of study, a greater effective flow $318 \pm 23 \mathrm{ml} / \mathrm{m}$ was observed in catheters with heparin lock compared to $307 \pm 17 \mathrm{ml} / \mathrm{m}$ with fibrilin $(p=0.008)$, lower venous pressure $147 \pm 12$ $\mathrm{mm} \mathrm{Hg}$ in heparin compared to $168 \pm 17$ in fibrilin $(p=0,006)$, higher KT in heparin $43 \pm 3$ litres compared to $41 \pm 4$ litres in fibrilin. Despite these better conditions, they did not represent clinical differences in dialysis efficacy, KT/V heparin $1.56 \pm 0.2$ compared to $1.59 \pm 0.2$ in fibrilin. A higher number of catheter handlings was observed in heparin $12 \pm 0.2$ compared to $9.4 \pm 1.3$ in fibrilin $(p=0.001)$. There were no differences in the appearance of infections, recirculation, need for fibrinolytics or coagulation alterations.

We conclude that the use of fibrilin lock solution for haemodialysis catheters is an effective alternative to $5 \%$ heparin. It is not accompanied by a higher degree of catheter dysfunction but does involve less handling, which could lead to fewer catheter-associated infections.

\section{KEY WORDS:}

- LOCK

- CATHETER

- $5 \%$ HEPARIN

- FIBRILIN

\section{Introducción}

Las soluciones de sellado de los catéteres son objeto común de debate entre los profesionales de nefrología. Recientemente y tras evaluar diversos estudios y distintos parámetros, la sociedad americana de nefrología intervencionista recomienda el empleo de heparina sódica al $1 \%$ (1000 unidades por mililitro) o citrato sódico al $4 \%{ }^{(1)}$. Recientemente Maki et al, estudió una solución de sellado compuesto por citrato sódico, metil y propil parabens y azul de metileno comparado con heparina sódica al $5 \%$, demostrando una eficacia superior en cuanto a una mayor reducción de infecciones, sin mostrar alteraciones en la función del catéter ${ }^{(2)}$.

La heparina sódica al 5\% es utilizada frecuentemente para el sellado del catéter a pesar de que existen estudios que demuestran un mayor $n^{\circ}$ de sangrados por exceso de heparinización ${ }^{(3)}$, sobre todo tras su colocación. Fibrilin es un producto compuesto por Heparina sódica $20 \mathrm{ui} / \mathrm{ml}$, cantidad recomendada en las guías de acceso vasculares de la $\mathrm{SEN}^{(4)}$, además de metil y propilparaben que ha demostrado ser útil como solución anticoagulante en el sellado de catéter sin producir anticoagulación sistémica, incluso cuando la solución sellante es introducida en la circulación sanguínea.

Ya que los problemas fundamentales asociados a la utilización de catéteres de Hemodiálisis son las infecciones y la disfunción del catéter, definida esta última como la imposibilidad de realizar una diálisis con un flujo>= de $300 \mathrm{ml} / \mathrm{m}^{(5)}$.

Nos planteamos como hipótesis de estudio que el Fibrilin® podía mantener una función del catéter no inferior a la heparina al $5 \%$.

Por ello nos fijamos como objetivo comparar dos soluciones de cebado heparina $\mathrm{Na}$ al $5 \%$ y Fibrilin ${ }^{\circledast}$, estudiando el funcionamiento del catéter y la eficacia de la diálisis, así como la aparición de infecciones y las necesidades de manipulación del catéter con cada una de las soluciones.

\section{Pacientes y métodos}

Se estudian 8 pacientes, mujeres, edad media $69 \pm 12$ años que realizan diálisis a través de catéter tunelizado en nuestra unidad ( $n^{\circ}$ total pacientes $=90$ ) en los que no se ha demostrado ningún tipo de disfunción de catéter en las dos últimas semanas y que firmen un consentimiento informado. Se desestiman aquellos pacientes con catéteres disfuncionantes, con catéteres temporales, o que refieran algún efecto adverso o alergia a algún componente de las soluciones.

En una paciente el catéter empleado es Cannon Catheter (Arrow) con un volumen de cebado de 2,2 el arterial 
y 2,4 el venoso y en siete pacientes se empleó el Split catéter III (Medcomp) con un volumen de cebado de 1,8 el arterial y 1,9 cc el venoso. Cinco catéteres estaban implantados en vena yugular interna ( 3 izquierdas y 2 derechas) y tres en vena subclavia (2 derechas y 1 izquierda).

Todos los pacientes se dializan en maquina Fresenius $4008 \mathrm{~S}$, con agua ultrapura y membrana Fx 80 . Se realiza heparinización durante la hemodiálisis con enoxaparina. Las dosis se mantuvieron invariables durante todo el estudio.

Se realizaron todas las conexiones según las normas de asepsia recomendadas en las guías SEN. Los tapones de conexión fueron $\mathrm{TEGO}{ }^{\circledR}$ en todos los pacientes, que se cambian por protocolo cada semana.

Inicialmente se sellaron los catéteres con FIBRILIN ${ }^{\circledR}$ (las ampollas contienen $5 \mathrm{ml}$ de la solución) que se repartirá en las dos luces del mismo. La solución no se aspira del catéter en la siguiente diálisis, si no que se conecta directamente al paciente.

Previamente a la conexión del paciente al circuito de diálisis se realizará un estudio de coagulación (el día intermedio de la semana) extraída de una vía periférica contralateral al catéter si es posible. Este estudio se realizará la primera semana del estudio y la sexta semana.

Se registrarán los siguientes parámetros: velocidad de bomba, recirculación, presión venosa y flujo efectivo. Episodios de poco flujo $(<300 \mathrm{ml} / \mathrm{m})$. Hipotensiones en diálisis. $\mathrm{N}^{0}$ de veces que se manipula el catéter. $\mathrm{T}^{\mathrm{a}} \mathrm{du}$ rante las diálisis. Episodios de tiritona. KT, KT/V. Necesidad de agentes fibrinoliticos.

Tras un mes de sellado con Fibrilin® (12 sesiones) se comienza el sellado con heparina al 5\% (12 sesiones). La cantidad de heparina para el sellado es la recomendada por el fabricante $+0,2 \mathrm{cc}$. Se realizarán las mismas medidas pero a diferencia del fibrilin ${ }^{\circledR}$ la heparina del catéter deberá aspirarse antes de iniciar la conexión al circuito de diálisis.

Se compararán los datos de ambos sellados por semana. Para los datos que se ajusten a una distribución normal se aplicará $t$ student en datos paramétricos y en los que no se ajuste a una distribución normal Man Withney. En datos no paramétricos el Chi cuadrado, test de Fisher y Wilcoxon. Análisis en SPSS 15.

\section{Resultados}

Las características basales se reflejan en la tabla $\mathbf{1}$. Se han estudiado 8 pacientes que son portadoras de un catéter en nuestra unidad ( $n^{\circ}$ total de pacientes $=90$ ).

\begin{tabular}{lcc}
\hline Variable & Media & Desv. Tip. (rango) \\
\hline Edad ( años) & 69,2 & $12(45-82)$ \\
\hline Peso ( kilos) & 64,5 & $13,4(49-92)$ \\
\hline Tiempo diálisis (meses) & 65 & $46(10-156)$ \\
\hline Duración catéter (meses) & 18 & $6,7(9-28)$ \\
\hline Duración diálisis (horas) & 3,4 & $0,3(3-4)$
\end{tabular}

Tabla 1. Características basales.

La velocidad de la bomba, flujo y KT fueron mayores en los pacientes que se sellaron con heparina $5 \%(p=0,01$, $p=0,008, p=0,001$ respectivamente). La presión venosa fue mayor en el grupo de pacientes sellados con fibrilin $(p=0,006)$. Estos resultados están reflejados en las tablas 2, 3, 4 y 5 . A pesar de estas diferencias, clínicamente no fueron relevantes; el flujo efectivo con fibrilin nunca fue inferior a $300 \mathrm{ml} / \mathrm{m}$ como marcador de disfunción y la presión venosa máxima registrada fue de $171 \mathrm{~mm} \mathrm{Hg}$ en la $3^{\circ}$ semana del estudio. El KT es 2,4 litros superior en el grupo de heparina sin significación clínica ya que el KT/V mediante Daugirdas fue similar en ambos grupos.

\begin{tabular}{|l|r|r|r|}
\hline & $\begin{array}{c}\text { FIBRILIN } \\
\mathbf{m l} / \mathbf{m}\end{array}$ & $\begin{array}{c}\text { HEPARINA } \\
\mathbf{m l} / \mathbf{m}\end{array}$ & \multicolumn{1}{|c|}{$\mathbf{P}$} \\
\hline Velocidad 1 semana & $342,12 \pm 20$ & $352,25 \pm 37$ & 0,2 \\
\hline Velocidad 2 semana & $337,25 \pm 16$ & $355 \pm 26$ & 0,02 \\
\hline Velocidad 3 semana & $338,75 \pm 21$ & $350,5 \pm 18$ & 0,11 \\
\hline Velocidad 4 semana & $344,12 \pm 29$ & $346,62 \pm 27$ & 0,7 \\
\hline Media velocidad de bomba & $340,56 \pm 20$ & $351,09 \pm 24$ & 0,01
\end{tabular}

Tabla 2. Velocidad de bomba del circuito.

\begin{tabular}{|c|c|c|c|}
\hline & $\frac{\text { FIBRILIN }}{\mathrm{ml} / \mathrm{m}}$ & $\frac{\text { HEPARINA }}{\mathrm{ml} / \mathrm{m}}$ & $\underline{\mathbf{P}}$ \\
\hline Flujo $1^{a}$ semana & $304,5 \pm 20$ & $316 \pm 33$ & 0,21 \\
\hline Flujo $2^{\circ}$ semana & $305,37 \pm 13$ & $320,75 \pm 25$ & 0,04 \\
\hline Flujo $3^{\circ}$ semana & $307,25 \pm 18$ & $322,12 \pm 22$ & 0,03 \\
\hline Flujo $4^{\circ}$ semana & $310,87 \pm 26$ & $317 \pm 17$ & 0,43 \\
\hline Media de flujo & $307 \pm 17$ & $318 \pm 23$ & 0,008 \\
\hline
\end{tabular}

Tabla 3. Flujo efectivo. 


\begin{tabular}{|l|c|c|c|}
\hline & $\frac{\text { FIBRILIN }}{\text { Litros }}$ & $\frac{\text { HEPARINA }}{\text { Litros }}$ & p \\
\hline KT 1 sem fib & $40,51 \pm 3$ & $41,85 \pm 4$ & 0,5 \\
\hline KT 2 sem fib & $41,57 \pm 4$ & $43,85 \pm 3$ & 0,03 \\
\hline KT 3 sem fib & $39,85 \pm 5$ & $43,42 \pm 3$ & 0,8 \\
\hline KT 4 sem fib & $41,28 \pm 5$ & $43,85 \pm 3$ & 0,007 \\
\hline Media KT Fibrilin & $40,8 \pm 4$ & $43,25 \pm 3$ & 0,001 \\
\hline
\end{tabular}

Tabla 4. KT

\begin{tabular}{|c|c|c|c|}
\hline & $\frac{\text { FIBRILIN }}{\mathrm{mm} \mathrm{Hg}}$ & $\frac{\text { HEPARINA }}{\mathrm{mm} \mathrm{Hg}}$ & $\mathbf{p}$ \\
\hline P Venosa $1^{\circ}$ sem & $165,25 \pm 30$ & $147,12 \pm 25$ & 0,1 \\
\hline P Venosa $2^{\circ}$ sem & $169,25 \pm 19$ & $146,25 \pm 17$ & 0,01 \\
\hline P Venosa $3^{\circ}$ sem & $171,5 \pm 23$ & $148,5 \pm 17$ & 0,04 \\
\hline P Venosa $4^{\circ}$ sem & $169,37 \pm 20$ & $149,12 \pm 8$ & 0.01 \\
\hline Media PV & $168,84 \pm 18$ & $147,75 \pm 12$ & 0,006 \\
\hline
\end{tabular}

Tabla 5. Presión venosa.

\begin{tabular}{|l|c|c|c|} 
& $\underline{\text { FIBRILIN }}$ & $\underline{\text { HEPARINA }}$ & $\underline{\mathbf{P}}$ \\
\hline Recirculación (\%) & $6,26 \pm 2,9$ & $6,01 \pm 2,3$ & 0,810 \\
\hline $\mathbf{N}^{0}$ manipulaciones & $9,41 \pm 1,3$ & $12,07 \pm 0,2$ & 0,001 \\
\hline Hipotensiones (\%) & $9,75 \pm 11$ & $14,37 \pm 18$ & 0,220 \\
\hline Tiempo protombina & $87,66 \pm 26$ & $88,11 \pm 16$ & 0,940 \\
\hline TPTA & $34,53 \pm 10$ & $34,25 \pm 8$ & 0,960 \\
\hline KT/V & $1,59 \pm 0,2$ & $1,56 \pm 0,2$ & 0,610 \\
\hline
\end{tabular}

Tabla 6. Otros parámetros

Aunque el porcentaje de hipotensiones fue algo mayor en el grupo de heparina, la diferencia no fue relevante. Si lo fue, en cambio el $n^{\circ}$ de manipulaciones, mayor en el grupo de heparina frente a Fibrilin: $12 \pm 02$ vs $9,4 \pm 1,3$; $p=0,001$. No existieron diferencias en el estudio de coagulación (TP, TPTA) en ambos grupos (Tabla 6). No se registraron infecciones asociadas al uso de catéteres y no fue necesario el uso de fibrinolíticos durante el período de estudio en ambos grupos.

\section{Discusión}

El sellado de catéteres para diálisis suele ser motivo de debate en la literatura y foros de discusión entre los profesionales de diálisis. En los últimos años distintos grupos publicaron distintos resultados con las soluciones que habitualmente son usadas para el sellado de los catéteres (suero salino, poligeli- na, heparina al $1 \%, 5 \%$, citrato trisódico al $37 \%$, $4 \%$, antibióticos, etc). Su finalidad iba encaminada a disminuir la tasa de infecciones, en algunos casos en detrimento de la función del mismo. La sociedad americana de nefrología intervencionista recomendó recientemente el uso de heparina al $1 \%$ o citrato al $4 \%{ }^{(1)}$ para evitar disfunción del catéter. Recientemente Maki et $\mathrm{al}^{(2)}$ estudiaron el efecto de una nueva solución sellante (Zuragen $\circledast$ ), compuesta de citrato al $4 \%$, parabens y azul de metileno y sus conclusiones fueron que disminuía el $\mathrm{n}^{\circ}$ de infecciones consiguiendo un flujo del catéter similar al de heparina, si bien la utilización de agentes fibrinoliticos fue mayor.

Se conoce poco sobre el uso de Fibrilin ${ }^{\circledR}$ en el sellado de catéteres en hemodiálisis ya que es usado generalmente para sellar otro tipo de catéteres. E. Gisbert comunicó la experiencia de su grupo de 18 pacientes durante 591 sesiones de diálisis con buen funcionamiento del catéter, menor $\mathrm{n}^{\circ}$ de manipulaciones, una menor carga de trabajo y menor coste ${ }^{(6)}$. Nuestro estudio difiere en que los catéteres son todos tunelizados, la dosis de Fibrilin es distinta, los pacientes recibieron los dos protocolos y el tiempo de seguimiento fue inferior. Aun así, los resultados son parecidos en cuanto la eficacia de la solución y menor $\mathrm{n}^{\circ}$ de manipulaciones.

Nuestro estudio es un pequeño estudio piloto que demuestra la no inferioridad de Fibrilin frente a heparina al 5\%. El estudio tiene algunas limitaciones, por lo que lo consideramos "piloto". No fue doble ciego dado que la solución de Fibrilin no se aspiraba y el tiempo de estudio es corto para demostrar un menor $n^{\circ}$ de infecciones como consecuencia de un menor $n^{\circ}$ de manipulaciones. Sería interesante hacer un estudio prospectivo, randomizado, controlado para demostrar esto último y además compararlo con otras soluciones del mercado como heparina al $1 \%$ o citrato.
Recibido: 10 Enero 2013

Revisado: 30 Enero 2013

Modificado: 20 Febrero 2013

Aceptado: 3 Marzo 2013 


\section{Bibliografía}

1. John E Moran, Stephen R Ash and the clinical practice committe Locking solutions for hemodialysis catheters: Heparin and citrate $-\mathrm{A}$ position paper by ASDIN. Seminars in dialysis. Vol. 21: 490 $-492 ; 2008$.

2. Dennis G Maki, Stephen R Ash, Roland K Winger, Philip Lavin, for the AZEPTIC Trial investigators. A novel antimicrobial and antithrombotic lock solution for hemodialysis catheters : A multicenter, controlled, randomized trial. Crit care 39(4):1 - 8; 2011.

3. Hüseyin Karaaslan, Pierre Peyronnet, Daniel Benevent, Christian Lagarde, Michel Rince, Claude
Leroux-Robert. Risk of heparin lock relate3d bleeding when using indwelling venous catheter in hemodialysis. Nephrol Dial Transplant 16: 2072 $2074 ; 2001$.

4. Guías de acceso vascular en Hemodiálisis. Guías SEN. Nefrología. Vol. XXV,Suplemento 1. 2005.

5. National Kindney Foundation: K/DOQI clinical practice guidelines for vadcular Access. Am J Kidney Dis 48:S248-S257;2006.

6. Elena Gisbert Rosique, $M^{\mathrm{a}}$ Carmen Pascual Cuadrado, Miguel Castillo Diaz. Estudio comparativo del sellado de catéteres con heparina sódica al 5\% o monodosis de heparina sódica de 20 ui/ml. Comunicación Congreso SEDEN (cd 1981-2010), Madrid 2011. 